\title{
Lefschetz pencils and mapping class groups
}

\author{
S. K. Donaldson
}

December 152004

\section{Introduction}

Holomorphic maps between complex manifolds have many properties which distinguish them among general smooth maps. Consider, for example, the case of a map between Riemann surfaces. A holomorphic map is represented locally, in suitable co-ordinates, by one of the models $z \mapsto z^{k}$ for $k \geq 0$. These models are very different from the models of generic smooth maps between surfaces, which are, in addition to the points where the map is a local diffeomorphism, folds and cusps. It is interesting to see what happens if we perturb the holomorphic map $z \mapsto z^{2}$ by a small non-holomorphic term. So for $\epsilon>0$ we define $f^{(\epsilon)}: \mathbf{C} \rightarrow \mathbf{C}$ by

$$
f^{(\epsilon)}(z)=z^{2}+2 \epsilon \bar{z}
$$

Thus $\frac{\partial f^{(\epsilon)}}{\partial z}=2 z$ and $\frac{\partial f^{(\epsilon)}}{\partial \bar{z}}=2 \epsilon$. The real derivative of $f^{(\epsilon)}$, has rank 2 at points where $\left|\frac{\partial f^{(\epsilon)}}{\partial z}\right| \neq\left|\frac{\partial f^{(\epsilon)}}{\partial \bar{z}}\right|$, that is to say where $|z| \neq \epsilon$. The point $z=\epsilon e^{i \theta}$ maps to the point $\gamma(\theta)=\epsilon^{2}\left(e^{2 i \theta}+2 e^{-i \theta}\right)$ and $\gamma^{\prime}(\theta)=2 i \epsilon^{2} e^{-i \theta}\left(e^{3 i \theta}-1\right)$. Thus $\gamma^{\prime}$ vanishes at the three points cube roots of unity. These three points are cusps of the map $f^{(\epsilon)}$ and the remaining points on the circle $|z|=\epsilon$ are fold points: the map $f^{(\epsilon)}$ maps this circle onto a curvilinear triangle with the three cusps as vertices. The reader is invited to visualise this map.

This example illustrates that holomorphic maps can be much simpler than typical smooth maps. We can abstract the local character of holomorphic maps and consider smooth maps which are locally modelled on holomorphic ones. This gives a way to study and exploit the topological aspects of holomorphic maps, independent of the finer details of the complex geometry and in a wider setting. For example we can consider branched covers of 2manifolds as a class of maps, independent of the existence of Riemann surface structures. A natural setting for this theory turns out to be symplectic topology, and the mapping class groups of surfaces, and certain generalisations, 
enter in an essential way. In this article we will outline parts of this theory and discuss some open problems. A discussion in a somewhat similar spirit will be found in [5].

\section{Holomorphic Morse theory and Dehn twists}

Let $f: X \rightarrow \mathbf{C}$ be a proper, nonconstant, holomorphic map from a connected complex manifold $X$ of complex dimension $n$ to the complex numbers. We suppose that $f$ is a "holomorphic Morse function", so that at each point where the derivative $\partial f$ vanishes the Hessian $\partial^{2} f$ is nondegenerate. These critical points form a discrete set in $X$. For simplicity we also suppose that the images of these points (the critical values) are distinct, so for each critical value there is just one corresponding critical point. Let $\Delta$ denote the set of critical values in C. Just as in ordinary Morse Theory, the essential thing in understanding the topology of the map $f$ is to understand the fibres $X_{t}=$ $f^{-1}(t)$ where $t \in \mathbf{C}$ is either a critical value or close to a critical value. The difference from the real case is that for $t \notin \Delta$ the fibres are all diffeomorphic. Indeed if we put $X^{\prime}=f^{-1}(\mathbf{C} \backslash \Delta)$ then the restriction $f: X^{\prime} \rightarrow \mathbf{C} \backslash \Delta$ is a $C^{\infty}$ fibration and the base $\mathbf{C} \backslash \Delta$ is connected (in contrast to the analogous situation in real geometry). Now recall the general notion of monodromy. Suppose $\phi: E \rightarrow B$ is a $C^{\infty}$ fibration over a connected base, with base point $b_{0} \in B$ and with fibre $F=\phi^{-1}\left(b_{0}\right)$. Let $\Gamma_{F}$ denote the mapping class group of the fibre: the isotopy classes of self-diffeomorphisms of $F$. Then we have a monodromy homomorphism

$$
\rho_{\phi}: \pi_{1}\left(B, b_{0}\right) \rightarrow \Gamma_{F}
$$

This can be defined by choosing a Riemannian metric on the total space $E$ which gives a family of horizontal subspaces: the orthogonal complements of the tangent spaces to the fibres. (In other language we can regard this as a choice of connection on the bundle $E$ regarded as a bundle with structure group $\operatorname{Diff}(F)$.) For any smooth based loop $\gamma:[0,1] \rightarrow B$ and any point $y$ in $\phi^{-1}\left(b_{0}\right)$ there is a horizontal lift $\tilde{\gamma}$ of $\gamma$ starting at $y$. We define $R: F \rightarrow F$ by $R(y)=\tilde{\gamma}(1)$. Then $R$ is a diffeomorphism of the fibre which, up to isotopy, is independent of the choice of metric and homotopy class of the loop $\gamma$.

Applied in our situation we get a monodromy homomorphism

$$
\rho_{f}: \pi_{1}(\mathbf{C} \backslash \Delta) \rightarrow \Gamma_{F}
$$

where $F$ is the fibre over some fixed base point in $\mathbf{C} \backslash \Delta$. Of course the fundamental group of $\mathbf{C} \backslash \Delta$ is a free group, with standard generators $\gamma_{i}$, 
say, winding once around a single critical value. This notion has two roots in classical complex analysis. On the one hand we can consider the case when $n=1$, so $X$ is a Riemann surface presented as a branched cover of C. The fibre $F$ is just a set of $d$ points, where $d$ is the degree of the map, and the mapping class group is the permutation group $\mathcal{S}_{d}$ on $d$ objects. The monodromy is just the data discussed in standard Riemann surface texts, which specifies how to glue together the sheets of the branched covering. In this case $\rho\left(\gamma_{i}\right)$ is a transposition in $\mathcal{S}_{d}$. On the other hand we can consider the case where $n=2$ so the fibres are Riemann surfaces. Thus $f: X \rightarrow \mathbf{C}$ can be regarded as a family of Riemann surfaces which degenerate over $\Delta$. The classical topic here is not so much the monodromy in the isotopy group but its composite with the natural action of the diffeomorphisms on the homology of the fibre, which yields a homological monodromy

$$
\rho_{f}^{H_{1}}: \pi_{1}(\mathbf{C} \backslash \Delta) \rightarrow G L\left(H_{1}(F)\right) .
$$

For example, let $z_{1}, \ldots, z_{n}$ be fixed distinct points in $\mathbf{C}$ and let $X_{0}$ be the subset of $\mathbf{C}^{3}$

$$
X_{0}=\left\{(z, w, \lambda): w^{2}=(z-\lambda)\left(z-z_{1}\right) \ldots\left(z-z_{n}\right)\right\} .
$$

Let $f_{0}$ be the restriction of the projection $(z, w, \lambda) \mapsto \lambda$. In a standard way, we can compactify the fibres of $f_{0}$ to obtain a complex manifold $X$, containing $X_{0}$ as a dense open set, and with a extension of $f_{0}$ to a proper holomorphic map $f: X \rightarrow \mathbf{C}$. Then the set $\Delta$ is just $\left\{z_{1}, \ldots, z_{n}\right\}$ and for $\lambda \notin \Delta$ the fibre $f^{-1}(\lambda)$ is the hyperelliptic Riemann surface defined by the equation

$$
w=\sqrt{(z-\lambda)\left(z-z_{1}\right) \ldots\left(z-z_{n}\right)} .
$$

The expression

$$
\frac{d z}{\sqrt{(z-\lambda) \ldots\left(z-z_{n}\right)}}
$$

defines a smoothly varying family of holomorphic 1-forms over the smooth fibres. Locally in the base we can fix a basis for the homology of the fibre and hence define the corresponding periods, by integrating the holomorphic form. Explicitly, this amounts to choosing a suitable collection of paths $\sigma_{\alpha}$ in $\mathbf{C}$ with end points in $\left\{z_{1}, \ldots, z_{n}, \lambda\right\}$ and the periods are then written as

$$
\int_{\sigma_{\alpha}} \frac{d z}{\sqrt{(z-\lambda) \ldots\left(z-z_{n}\right)}},
$$

for a choice of branch of the square root. The issue addressed by the knowledge of the monodromy homomorphism, in this special case, is how these 
contour integrals change when $\lambda$ traces out a path encircling one of the fixed points $z_{i}$.

We can now discuss the central issue: what is the monodromy of a holomorphic Morse function around a loop about a single critical value? This is the analogue of the description of the change in the level set of a real Morse function as one crosses a critical value. As in that case, a crucial observation is that the problem can be reduced to a standard local model. Indeed if we take a very small loop $\gamma_{i}$ about a critical value then we can choose the horizontal subspaces so that the monodromy is the identity map outside the intersection $F \cap B$ of the fibre $F$ with a suitable small ball $B$ in $X$ centred on the critical value. Then we can regard the monodromy as a compactly supported diffeomorphism of $F \cap B$, defined up to compactly supported isotopy. To see what is going on take the standard local model to be the map

$$
g\left(z_{1}, \ldots, z_{n}\right)=z_{1}^{2}+\ldots+z_{n}^{2},
$$

from $\mathbf{C}^{n}$ to $\mathbf{C}$, and take the base point $b_{0}$ to be $1 \in \mathbf{C}$. We consider the subset of the fibre $g^{-1}\left(b_{0}\right)$ given by the real points $g^{-1}\left(b_{0}\right) \cap \mathbf{R}^{n}$ which we denote by $V$. This is just the standard unit sphere in $\mathbf{R}^{n}$. For any $t \in \mathbf{R}$ we can consider similarly $g^{-1}(t) \cap \mathbf{R}^{n}$. This is the sphere of radius $\sqrt{t}$ for $t \geq 0$ and the empty set if $t<0$. We can choose a family of horizontal subspaces which preserves the real points so if we "parallel transport" the fibre from 1 towards 0 along the positive real axis the parallel transports of $V$ shrink down to the critical point.

Now consider the total space $T S^{n}$ of the tangent bundle of the $n$-sphere. The standard "generalised Dehn twist" is a compactly supported diffeomorphism of $T S^{n}$, canonical up to compactly supported isotopy. We can define it as follows. The points in $T S^{n}$ can be identified with pairs $(v, w)$ of orthogonal vectors $v, w$ in $\mathbf{R}^{n}$, where $|v|=1$. If $w \neq 0$ then for any angle $\theta$ we define the usual rotation $R_{\theta}$ in the (oriented) plane spanned by $v, w$. Now choose a function $\Theta$ on $[0, \infty)$ such that $\Theta(s)=0$ if $s$ is small and $\Theta(s)=\pi$ if $s$ is large. Define a map $D: T S^{n} \rightarrow T S^{n}$ by

$$
D(v, w)=R_{\Theta(|w|)}(-v,-w),
$$

with the obvious interpretation if $w=0$ : i.e. $D(v, 0)=(-v, 0)$. Then $D(v, w)=(v, w)$ if $|w|$ is large and $D(v, w)=(-v,-w)$ if $|w|$ is small. Also, $D$ is a diffeomorphism, with inverse

$$
D^{-1}\left(v^{\prime}, w^{\prime}\right)=R_{-\Theta\left(\left|w^{\prime}\right|\right)}\left(-v^{\prime},-w^{\prime}\right) .
$$

Thus this model Dehn twist is a compactly supported diffeomorphism of $T S^{n}$, equal to the antipodal map on the zero section. 
Now return to the sphere $V \subset g^{-1}\left(b_{0}\right)$ in the standard model above. This sphere is a "totally real" submanifold of the fibre; that is, multiplication of tangent vectors by $I$ yields an identification between the tangent bundle $T V$ and the normal bundle of $V$ in the fibre $F=g^{-1}\left(b_{0}\right)$. In other words, a tubular neighbourhood $N$ of $V$ in $F$ can be identified with a neighbourhood $N^{\prime}$ of the zero section in the tangent bundle of $V$. Now we can obviously suppose that our model map $D$ is supported in $N^{\prime}$ and so, via this identification, we can regard $D$ as a diffeomorphism of $F$, supported in the neighbourhood $N^{\prime}$. The fundamental fact is that this is the monodromy around the critical value.

In general then we arrive at the following description of the topology of a map $f: X \rightarrow \mathbf{C}$ as considered above.

- In the model fibre $F=f^{-1}\left(b_{0}\right)$ there are "vanishing cycles" $V_{i}$ associated to the critical values $z_{i}$ (and the loops $\gamma_{i}$ around the $z_{i}$ ). Each $V_{i}$ is an embedded $(n-1)$-sphere uniquely defined up to isotopy, and we have an identification (fixed up to homotopy) of the normal bundle of $V_{i}$ in $F$ with the tangent bundle.

- The monodromy around the loop $\gamma_{i}$ is the Dehn twist $D_{V_{i}}$ about $V_{i}$, defined using an identification of a tubular neighboourhood as above (which is independent of choices, up to isotopy).

We can relate this discussion to the classical problems considered above. First, if $n=1$ the "vanishing cycle" is just a copy of $S^{0}$, i.e. a pair of points, and the "Dehn twist" is just a transposition (the antipodal map on $S^{0}$ ). If $n=2$ we get the familiar Dehn twists in the mapping class groups of Riemann surfaces. It is easy to see that the Dehn twist about an embedded circle $V$ in a Riemann surface $\Sigma$ acts on $H_{1}(\Sigma)$ by

$$
\alpha \mapsto \alpha+\langle V, \alpha\rangle V
$$

where $\langle$,$\rangle is the intersection form. So we arrive at the classical Picard-$ Lefschetz formula for the homological monodromy $\rho^{H_{1}}$.

Some words about signs may be in order here. Given an embedded circle $V$ in a 2-manifold $\Sigma$, the Dehn twist about $V$ is completely specified by a choice of orientation of $\Sigma$, it does not require an orientation of $V$. Thus in the Picard-Lefschetz formula (1) we have momentarily fixed an orientation of $V$, to define the homology class, but obviously the formula is unchanged if we change orientation. On the other hand, with a fixed orientation of $\Sigma$, the inverse of a Dehn twist is not a Dehn twist as we have defined things: it is a Dehn twist of the manifold $\Sigma$ with the opposite choice of orientation. The same holds in higher dimensions. Note however that the usual orientation of the neighbourhood of $V$ induced by the identification with $T V$ differs 
from the standard complex orientation by a sign $(-1)^{n-1}$. Thus when $n$ is odd the self-intersection of $V$, with respect to the complex orientation is -2 . The Picard-Lefschetz formula (1) for the action on the middle-dimensional homology is the same in all dimensions but the significance is somewhat different depending whether $n$ is or even or odd. The fibre $F$ has real dimension $2(n-1)$ and the intersection form is antisymmetric if $n$ is even and symmetric if $n$ is odd. In the second case the Picard-Lefschetz transformation is of order 2 ; the reflection defined by vector $[V]$ with $[V] .[V]=-2$.

In the case when $n=1$ and $X$ is a 2-manifold we can reverse the constructions above. That is we have the classical

Proposition 1 There is a one-to-one correspondence between equivalence classes of data:

- Riemann surfaces $X$ with a proper map $f: X \rightarrow \mathbf{C}$ of degree $d \geq 1$ having only simple branch points, mapping to distinct points in $\mathbf{C}$.

- Discrete sets $\Delta \subset \mathbf{C}$ and homomorphisms $\rho: \pi_{1}(\mathbf{C} \backslash \Delta) \rightarrow \mathcal{S}_{d}$ mapping each standard generator to a transposition.

(We leave it to the reader to spell out the exact equivalence relations to impose on these two kinds of data.)

The analogue of this in higher dimensions does not hold: we cannot create a pair $(X, f)$ to realise arbitrary data $(\Delta, \rho)$. But we can extract the topology from the situation by enlarging our class of spaces.

Definition 1 A Topological Lefschetz Fibration consists of a smooth oriented $2 n$-dimensional manifold $X$ and a proper map $f: X \rightarrow \mathbf{C}$ with the following properties.

- For each point $x_{0}$ of $X$ either $d f_{x_{0}}$ is surjective or, when $x_{0}$ is a critical point of $f$, there is an oriented chart $\psi: U \rightarrow \tilde{U}$ where $U$ is a neighbourhood of $x_{0}$ in $X$ and $\tilde{U}$ is a neighbourhood of the origin in $\mathbf{C}^{n}$ such that the composite $\tilde{f}=f \circ \psi^{-1}$ is

$$
\tilde{f}\left(z_{1}, \ldots, z_{n}\right)=f\left(x_{0}\right)+z_{1}^{2}+\ldots+z_{n}^{2} .
$$

- If $x_{0}$ and $x_{1}$ are two different critical points of $f$ then $f\left(x_{0}\right) \neq f\left(x_{1}\right)$.

Clearly such a map has a well-defined smooth fibre $F$, an oriented 2(n-1)manifold.

We say that two Topological Lefschetz Fibrations $f_{1}: X_{1} \rightarrow \mathbf{C}, f_{2}$ : $X_{2} \rightarrow \mathbf{C}$ are are equivalent "over a fixed base" if there is a diffeomorphism 
$\alpha: X_{1} \rightarrow X_{2}$ with $f_{1}=f_{2} \circ \alpha$. This implies that the two fibrations have the same set of critical values in $\mathbf{C}$. Now fix a discrete subset $\Delta \subset \mathbf{C}$ and a set of standard generators $\gamma_{i}$ for $\pi_{1}(\mathbf{C} \backslash \Delta)$. Then we have

Proposition 2 There is a one-to one correspondence between:

- Topological Lefschetz fibrations $f: X \rightarrow \mathbf{C}$ with fibre $F$ and critical set $\Delta$ modulo equivalence over a fixed base.

- Collections of isotopy classes of embedded $(n-1)$-spheres $V_{i} \subset F$ with homotopy classes of isomorphisms between the normal and tangent bundles of the $V_{i}$, modulo equivalence induced by the action of a single element of $\Gamma_{F}$.

The proof of the Proposition is straightforward. Suppose for example that we are given data of the second kind. Let $\Omega$ be the complement in $\mathbf{C}$ of small disjoint discs about the points of $\Delta$. Then $\Omega$ is homotopy equivalent to a wedge of circles so by standard theory fibrations with fibre $F$ over $\Omega$ are determined by their monodromy. So we can construct a fibration $X_{\Omega} \rightarrow \Omega$ with the Dehn twists in the $V_{i}$ as monodromy around $\gamma_{i}$. On the other hand, given a single $V \subset F$, we can construct a standard model $\pi: Y_{V} \rightarrow \mathbf{C}$ whose monodromy around a large circle is the Dehn twist about $V$. Now we construct $X$ by gluing these standard models to $X_{\Omega}$ to fill in the missing discs.

The following example shows that one does need to take some care in formulating this correspondence. Suppose $n=2$ and $f: X \rightarrow \mathbf{C}$ is, say, a holomorphic Lefschetz fibration and $x_{0}$ is a point in $X$ with $f\left(x_{0}\right) \notin \Delta$. Now let $\hat{X}$ be the "blow-up" of $X$ at the point $x_{0}$ and $\hat{f}$ be the composite of the $f$ and the canonical map $\hat{X} \rightarrow X$. The critical set $\hat{\Delta}$ is $\Delta \cup\left\{f\left(x_{0}\right)\right\}$. The smooth fibres of $\hat{f}$ are the same as those of $f$. The fibre of $\hat{f}$ over $f\left(x_{0}\right)$ is the union of the smooth fibre $F$ and the exceptional sphere $E \cong S^{2}$, meeting at one point. What happens is that the identification between the fibres of $f$ and $\hat{f}$ realises the diffeomorphism beween the connected sum $F \sharp S^{2}$ and $F$. The vanishing cycle associated to the extra critical value is a trivial circle in $F$ (i.e it bounds a disc) and the resulting monodromy is trivial, up to isotopy. So $f: X \rightarrow \mathbf{C}$ and $\hat{f}: \hat{X} \rightarrow \mathbf{C}$ have in sense the "same" monodromy, even though the manifolds $X, \hat{X}$ are different.

There is another natural notion of equivalence between Topological Lefschetz fibrations. We say that $f_{1}: X_{1} \rightarrow \mathbf{C}, f_{2}: X_{2} \rightarrow \mathbf{C}$ are equivalent if there is a diffeomorphism $\alpha: X_{1} \rightarrow X_{2}$ and a diffeomorphism $\beta: \mathbf{C} \rightarrow \mathbf{C}$ such that $\beta \circ f_{1}=f_{2} \circ \alpha$. This means that $\beta$ maps the set of critical values of $f_{1}$ to that of $f_{2}$. Clearly the equivalence classes of Topological Lefschetz 
fibrations correspond to orbits of an a action of the group of diffeomorphisms of the plane with a marked set $\Delta$ on the set of equivalence classes over a fixed base.

\section{$3 \quad$ Lefschetz pencils and symplectic four-manifolds}

Holomorphic Lefschetz fibrations typically arise in the following way. For simplicity we will restrict the discussion to complex dimension 2. Suppose that $Y \subset \mathbf{C P}^{N}$ is a complex projective surface. We choose a generic $(N-2)$ dimensional subspace $\mathbf{C P}^{N-2} \subset \mathbf{C P}^{N}$, meeting $Y$ transversely in a finite set of points $A \subset Y$, and we consider the "pencil" of hyperplanes through $\mathbf{C P}^{N-2}$. These cut out a 1-parameter family of hyperplane sections of $Y$. Suppose $Z_{i}$ are homogeneous co-ordinates on $\mathbf{C P}^{N}$ and $\mathbf{C} \mathbf{P}^{N-2}$ is defined by the equations $Z_{0}=Z_{1}=0$. Then $g=Z_{1} / Z_{0}$ is a meromorphic function on $Y$ and the hyperplane sections are the fibres $g^{-1}(\lambda)$, for different $\lambda \in \mathbf{C P}^{1}$. The meromorphic function $g$ is not a well-defined map on $Y$ but if we blow up the points of $A$ we get a well-defined map $\tilde{g}: X \rightarrow \mathbf{C P}^{1}$ where $X$ is the blow up of $Y$. For generic choices of the axis $\mathbf{C P}^{N-2}$ the restriction of $\tilde{g}$ to $\tilde{g}^{-1}(\mathbf{C})$ will be a holomorphic Lefschetz fibration of the kind considered before. Thus our previous discussion needs to be extended in two ways

- In place of the base $\mathbf{C}$ we have the Riemann sphere $\mathbf{C P}^{1}$. This makes little difference. We can arrange things so that the fibre over $\infty$ is smooth, then we have the additional constraint that the product of the monodromies around all the $\gamma_{i}$ should be trivial regarded as an element of the mapping class group of the fibre (since it represents the monodromy around a large circle in $\mathbf{C}$ ).

- We may wish to remember the fact that the total space $X$ arose as the blow up of $Y$. The exceptional spheres created by the blowing up appear as sections of the fibration. Thus we can take our fibre to be a Riemann surface $\Sigma$ with a collection of marked points $P=\left\{p_{\alpha}\right\}$, and our vanishing cycles to be circles in $\Sigma \backslash P$. We introduce the mapping class group $\Gamma_{\Sigma, P}$ of compactly supported diffeomorphisms of $\Sigma \backslash P$ modulo compactly supported isotopy. (Equivalently, diffeomorphims equal to the identity on fixed small discs about the $p_{\alpha}$.)Then the condition that the sections have self-intersection -1 goes over to the condition that

$$
D_{V_{1}} \circ \ldots D_{V_{\nu}}=T
$$

in $\Gamma_{\Sigma, P}$ where $T$ is the product of Dehn twists about small circles around 
the $p_{\alpha}$. (Thus $T$ is trivial in the unrestricted mapping class group, but not usually in $\Gamma_{\Sigma, P}$.)

Again we can define a topological analogue of this picture.

Definition 2 A Topological Lefschetz pencil (TLP) on a compact smooth oriented 4-manifold $X$ consists of the following data.

- Finite, disjoint subsets $A, B \subset X$.

- A smooth map $f: X \backslash A \rightarrow S^{2}$ which is a submersion outside $A \cup B$; such that $f(b) \neq f\left(b^{\prime}\right)$ for distinct $b, b^{\prime} \in B$ and which is given in suitable oriented charts by the local models $\left(z_{1}, z_{2}\right) \mapsto z_{2} / z_{1}$ (in a punctured neighboourhood of a point in $A$ ) and $\left(z_{1}, z_{2}\right) \mapsto z_{1}^{2}+z_{2}^{2}+$ Constant (in a neighbourhood of a point in $B$ ).

The notions of "equivalence over a fixed base" and "equivalence" go over in an obvious way.

Given a Topological Lefschetz pencil we define the "hyperplane class" $h \in H^{2}(X ; \mathbf{Z})$ to be the Poincaré dual of the homology class of a fibre of $f$. (More precisely, the closure in $X$ of a fibre of $f$ in $X \backslash A$.) We define another class $K(X, f) \in H^{2}(X ; \mathbf{Z})$ as follows. Over $X \backslash(A \cup B)$ we have an oriented 2-plane bundle $V$ given by the tangent space to the fibre of $f$. We claim that this can be extended to $X$ and the extension is unique up to isomorphism. For if $N$ is a small ball around a point of $A$ or $B$ we can extend $V$ over $N$ if we have a trivialisation of $V$ over $\partial N$. Since $\partial N \cong S^{3}$ and $H^{2}\left(S^{3}\right)=0$ such a trivialisation exists. Morover since $H^{1}\left(S^{3}\right)=0$ any two trivialisations are homotopic and this implies that any two extensions are isomorphic. Now we set

$$
K(X, f)=-\left(c_{1}(V)+2 f^{*}\left(\left[S^{2}\right]\right),\right.
$$

where $c_{1}(V)$ is the first Chern class of $V$, regarded as a complex line bundle.

By straightforward algebraic topology we have:

Proposition 3 - The genus of a smooth fibre of $f$ is $\frac{1}{2}(h . h+K(X, f) . h+$ 2).

- The number of points in $A$ is h.h.

- The number of points in $B$ is $\chi(X)+h . h+2 K(X, f) . h$.

So far we have been considering maps from either complex algebraic manifolds (the classical case) or general smooth manifolds. The interest of these 
ideas is highlighted by the connection with the intermediate class of symplectic manifolds. We say that a TLP on a 4-manifold $X$ is compatible with a symplectic form $\omega$ on $X$ if we can choose the local co-ordinates $\left(z_{1}, z_{2}\right)$ appearing in the definition such that $\omega$ is a Kahler form in these co-ordinates (i.e. has type $(1,1))$ and if the fibres of $f$ in $X \backslash(A \cup B)$ are oriented symplectic submanifolds (i.e $\omega$ is strictly positive on the fibre, with respect to the induced orientation). In general, for any symplectic manifold $(X, \omega)$ we can define a "canonical class" $K(\omega)$ to be minus the first Chern class of the tangent bundle, for any compatible almost complex structure. It is easy to see that if a pencil is compatible with a symplectic structure then $K(\omega)=K(X, f)$.

Theorem 1 Let $X$ be a smooth oriented 4-manifold and let $h \in H^{2}(X ; \mathbf{Z})$ be a class with $h^{2}>0$. Then if $X$ has a TLP with hyperplane class $h$ it admits a compatible symplectic form $\omega$ with $[\omega]=h$. Conversely if $X$ admits a symplectic form with $[\omega]=h$ then for sufficiently large integers $k, X$ admits a compatible TLP with hyperplane class $k h$.

This is a composite of results of Gompf [7] and the author [4]. The interest of the result is that the question of the existence of symplectic forms is, on the face of it, a question on the borderline of differential geometry and differential topology, while the question of the existence of TLP's is, on the face of it, pure differential topology. Thus the result is a topological criterion for the existence of symplectic structures on 4-manifolds.

We will not go into the proofs of this Theorem. In one direction, Gompf's construction of a symplectic form on a manifold admitting a Lefschetz pencil is an extension of Thurston's construction of symplectic forms on fibre bundles. The proof in the other direction involves some analysis. Roughly, one considers for large $k$ embeddings $\iota: X \rightarrow \mathbf{C P}^{N}$ with $\iota^{*}([H])=k[h]$ where $H$ is the standard generator of $H^{2}\left(\mathbf{C P}^{N}\right)$. One shows that, for any fixed compatible almost-complex structure on $X$, the embedding can be chosen to be "approximately holomorphic". This can be seen as an extension of the Kodaira embedding theorem from complex geometry to the almost-complex case. Then one constructs the pencil by choosing a sufficiently generic $\mathbf{C} \mathbf{P}^{N-2}$ and following the procedure described above.

\section{4 "Explicit" description of symplectic four- manifolds}

The discussion of the uniqueness of the TLP corresponding to a sympletic form is a little more complex, partly because there is a lacuna of a rather 
technical nature in the foundational results proved up to now. The problem is that in the analytical theory one is lead to the notion of an "asymptotic sequence" of TLP's $f_{k}$, defined for large $k$. As the theory stands, the precise definition of this notion would be rather complicated: it would involve the choice of an almost complex structure and various real parameters measuring roughly "deviation from holomorphicity" and "transversality". However we can formulate our results without going into these details, making the existence of the notion part of the statement.

Theorem 2 Let $(X, \omega)$ be a compact symplectic 4-manifold with $[\omega]$ integral and let $h$ be an integer lift of $[\omega]$. There is a preferred non-empty class $\mathcal{A}$ of sequences $\left(f_{k}\right)$ of TLP's on $X$, defined for large $k$ and where $f_{k}$ has hyperplane class $k h$, such that if $\left(f_{k}^{\prime}\right)$ is another sequence in the class $\mathcal{A}$ then $f_{k}$ is equivalent to $f_{k}^{\prime}$ for large $k$.

The issue that is left open here is that given some TLP $g$ it is hard to decide if $g$ is a member of an asymptotic sequence (although it seems quite likely that it will be except perhaps in some special circumstances).

Next we will describe the "stabilisation operation" due to Auroux and Katzarkov [1]. Suppose that $f$ is a TLP on $X$. Recall that this is determined by a smooth fibre $\Sigma$ with marked points $p_{\alpha}$ and vanishing cycles $V_{i} \subset \Sigma \backslash\left\{p_{\alpha}\right\}$ such that the product of the Dehn twists about the $V_{i}$ is equal to the element $T$ (the product of Dehn twists in circles about the $p_{\alpha}$ ) in the marked mapping class group $\Gamma_{\Sigma, P}$. We can think of $\Sigma$ as having fixed charts around the $p_{\alpha}$. Using these charts we form a new surface $\hat{\Sigma}$ by taking a second copy of $\Sigma$ and performing the connected sum operation at each of the points $p_{\alpha}$. Thus the genus of $\hat{\Sigma}$ is twice the genus of $\Sigma$ plus the number of points $p_{\alpha}$ minus 2. We fix a standard embedding $j$ of $\Sigma$ minus small discs about the $p_{\alpha}$ into $\hat{\Sigma}$. We take four standard points on each of the cylinders making up the connected sum, giving a set $\hat{P}$ of $4|P|=(2 h)^{2}$ marked points in $\hat{\Sigma}$. Now we work in the mapping class group $\Gamma_{\hat{\Sigma}, \hat{P}}$. Here we have the element $\hat{T}$ defined by the product of the Dehn twists in small loops about the points of $\hat{P}$ and we also have the element $T$ defined by the twists in the original loops about the $p_{\alpha}$, regarded as loops in $\hat{\Sigma}$ via the embedding $j$. Auroux and Katzarkov write down two explicit and standard collections of loops $\left\{U_{i}\right\},\left\{W_{i}\right\}$ in $\hat{\Sigma}$ such that

$$
\hat{T}=A T B
$$

in $\Gamma_{\hat{\Sigma}, \hat{P}}$ where $A$ is the product of Dehn twists in the $U_{i}$ and $B$ is the product of Dehn twists in the $W_{i}$. Now suppose that $V_{1}, \ldots, V_{\nu}$ are loops representing the monodromy of the pencil $f$. We can consider them as loops in $\hat{\Sigma}$, via the 
embedding $j$, and we have

$$
\hat{T}=A D_{V_{1}} \ldots D_{V_{\nu}} B=D_{U_{1}} \ldots D_{U_{p}} D_{V_{1}} \ldots D_{V_{\nu}} D_{W_{1}} \ldots D_{W_{q}} .
$$

So the $U_{i}, V_{i}, W_{i}$ are data defining a TLP with fibre $\hat{\Sigma}$. We call this TLP $S q(f)$.

Proposition 4 The 4-manifold associated to $S q(f)$ is diffeomorphic to $X$.

Theorem 3 [1] Let $f_{k}$ be a sequence in $\mathcal{A}$ associated to a symplectic 4manifold $(X, \omega)$. Then for large enough $k$, the TLP $f_{2 k}$ is equivalent to $S q\left(f_{k}\right)$.

The upshot of the results of the previous section is that in principle questions about the classification of symplectic 4-manifolds can be translated into questions about Dehn twists in the mapping class group of surfaces. Let us spell this out in more detail. Define a primitive compact symplectic 4 -manifold to be a triple $(X, h, \omega)$ where $X$ is compact 4-manifold, $h$ is a primitive integral cohomology class and $\omega$ is a symplectic form on $X$ with $[\omega]=h$. We give a description of the equivalence classes (in the obvious sense) of such primitive symplectic 4-manifolds.

We have explained that a TLP is specified by data consisting of loops $V_{i}$ in a marked surface $(\Sigma, P)$ satisfying the condition $D_{V_{1}} \ldots D_{V_{\nu}}=T^{-1}$. Now we ask when two such sets of data yield equivalent TLP's. If we just consider equivalence over a fixed base we have to consider the loops $V_{i}$ up to isotopy and modulo the action of conjugation of the $D_{V_{i}}$ by a single arbitrary element of $\Gamma_{\Sigma, P}$, i.e. changing each of the $V_{i}$ to $g\left(V_{i}\right)$ for some $g \in \Gamma_{\Sigma, P}$. If we consider general equivalence we have to bring in the action of the diffeomorphisms of the 2-sphere with $\nu$ marked points, the spherical braid group. It is convenient to regard the point at infinity as fixed, so we work with the group of compactly supported diffeomorphisms of the plane with $\nu$ marked points. This is just the Braid group $B_{\nu}$ and has standard generators $\sigma_{i}, i=1, \ldots \nu-1$. The action of $\sigma_{i}$ on the Dehn twists takes a sequence $D_{V_{1}}, \ldots, D_{V_{\nu}}$ to a sequence

$$
D_{V_{1}}, \ldots, D_{V_{i+1}}, D_{V_{i}}^{*}, \ldots, D_{V_{\nu}}
$$

where $D_{V_{i}}^{*}$ is the conjugate of $D_{V_{i}}$ :

$$
D_{V_{i+1}}^{-1} D_{V_{i}} D_{V_{i+1}} .
$$

Note that this action preserves the product, as it should. In other words we can change a sequence of embedded circles

$$
V_{1} \ldots V_{i}, V_{i+1}, \ldots, V_{\nu}
$$


to a new sequence

$$
V_{1} \ldots V_{i+1} V_{i}^{*} \ldots V_{\nu}
$$

where $V_{i}^{*}$ is obtained by applying the inverse Dehn twist in $V_{i+1}$ to $V_{i}$. Now for each $g, \nu, p$ we write $\mathcal{D}_{g, \nu, p}$ for the class of finite sequences of isotopy classes of circles $V_{1}, \ldots, V_{\nu}$, in a standard surface of genus $g$ with $p$ marked points, which satisfy the relation (2). So for each such sequence we can construct a 4-manifold with a TLP. Let $\mathcal{D}_{g, \nu, p}^{(0)}$ be the subclass consisting of such data which define a TLP where the class $F$ is $2^{r}$ times a primitive class, for some $r$. Now let $\mathcal{C}_{g, \nu, p}$ be the quotient of $\mathcal{D}_{g, \nu, p}^{(0)}$ under the action of the braid group and the mapping class group of $\left(\Sigma, p_{\alpha}\right)$. We have the Auroux-Katzarkov map

$$
S q: \mathcal{C}_{g, \nu, p} \rightarrow \mathcal{C}_{\hat{g}, \hat{\nu}, \hat{p}}
$$

where $\hat{g}=2 g+\nu-1, \hat{\nu}=4(g+\nu-1), \hat{p}=4 p$. Now if we write $\chi=\nu-2(2 g-$ $2), \theta=\frac{2 g-2-p}{\sqrt{p}}$ then we have, in an obvious notation, $\hat{\chi}=\chi, \hat{\theta}=\theta$. For fixed $\chi, \theta$ we let $\mathcal{C}_{\chi, \theta}$ be the direct limit of the maps of sets $S q: \mathcal{C}_{g, \nu, p} \rightarrow \mathcal{C}_{\hat{g}, \hat{p}, \hat{\nu}}$ with $\chi=\nu-2(2 g-2), \theta=\frac{2 g-2-p}{\sqrt{p}}$.

Now let $\mathcal{X}_{\chi, \theta}$ be the set of equivalence classes of data of the form $(X, \omega, h)$ where $X$ is a smooth compact 4-manifold of Euler characteristic $\chi, h$ is a primitive class in $H^{2}(X ; \mathbf{Z}), \omega$ is a symplectic form on $X$ with $[\omega]$ the reduction of $h$ and

$$
\frac{K(\omega) \cdot h}{\sqrt{h \cdot h}}=\theta .
$$

Then the results of the previous section amount to

Proposition 5 There is a canonical inclusion of $\mathcal{X}_{\chi, \theta}$ into $\mathcal{C}_{\chi, \theta}$

It seems most likely that this inclusion is in fact a bijection. This issue is the technical lacuna in the theory referred to above, i.e. the question whether any TLP, perhaps after stabilisation by the Auroux-Katzarkov construction, arises as an element of an asymptotic sequence. Assuming this is so we see that the problem of classifying compact symplectic 4-manifolds (with integral symplectic form and a chosen lift $h$ ) is equivalent to the problem of describing the set $\mathcal{C}_{\chi, \theta}$ which is formulated entirely in terms of the mapping class group

\section{Problems}

The significance of the translation of symplectic 4-manifold theory into problems about the mapping class group should not be overrated. In practice, the problem of classifying the appropriate sequences of embedded circles modulo equivalence seems very intractable and it may be unlikely that much can be 
done without some new idea. The situation is quite similar to that arising from the description of 3-manifolds via Heegard decompositions where again the apparent simplicity of the translated problem is largely illusory because of the complexity of the mapping class group. So far, no progress has been made in, for example, distinguishing symplectic 4-manifolds via this "combinatorial" approach. (Another way of seeing that this approach is unlikely to be immediately useful is that the classification problem, without restriction, contains the problem of classifying finitely presented groups, since any group arises as the fundamental group of a symplectic 4-manifold [7].) Nevertheless, we can state as an ambitious problem:

Problem 1 Develop techniques to describe the sets $\mathcal{C}_{\chi, \theta}$.

As we have said, the real difficulty comes from the action of the braid group. One can think of this in the following way. Make a directed graph with one vertex for each element of the mapping class group $\Gamma_{\Sigma, P}$ and one oriented edge joining $g$ and $g \circ D_{V}$ for each isotopy class of embedded circle $V$ in $\Sigma \backslash P$. Thus a sequence of $V_{1} \ldots V_{\nu}$ of the kind we want to consider is the same thing as an oriented path in the graph from the identity to the element $T$. Now for each vertex $g$ and pair $V_{1}, V_{2}$ we have four edges

$$
\left(g, g D_{V_{1}}\right),\left(g D_{V_{1}}, g D_{V_{1}} D_{V_{2}}\right),\left(g, g D_{V_{2}}\right),\left(g D_{V_{2}}, g D_{V_{1}} D_{V_{2}}\right)
$$

since $D_{V_{1}} D_{V_{2}}=D_{V_{2}} D_{V_{1}}^{*}$ where $D_{V_{1}}^{*}$ is the conjugate as before. Now form a topological space $Z$ by attaching a square to each such collection of four edges. Thus our braid relation is the relation on edge-paths in $Z$ under which we are allowed to push a path across a square in the obvious way. This is somewhat similar to the usual combinatorial description of the homotopy classes of paths in $Z$ from 1 to $T^{-1}$-i.e. essentially of $\pi_{1}(Z)$-but with the crucial difference that we are only allowed to consider "positive" paths. It seems likely that $\pi_{1}(Z)$ is just the integers: this would correspond to the fact that the description of TLP's becomes much simpler if we are allowed to use both positive and negative Dehn twists and cancel positive and negative pairs. Of course in formulating things this way we have not really done anything beyond restating the problem, but the point of view might be worth investigating. One could also try to fit the recent work of Auroux, Munoz and Presas [2], describing symplectomorphisms in terms of pencils, into the same mould, perhaps giving a model for the classifying space of the group of symplectomorphisms of a symplectic 4-manifold. Another related point of view is to think of a Lefschetz pencil as defining a map from the two sphere into the compactified moduli space $\overline{\mathcal{M}}_{g, P},[12]$. Again the problem is related to a standard one, of describing $\pi_{2}\left(\overline{\mathcal{M}}_{g, P}\right)$, but the real difficulty comes from the fact that we have to consider an equivalence defined by homotopies 
through maps which meet the compactification divisor with positive local intersection numbers.

Notice that the essence of the definition of $\mathcal{C}_{g, p, \nu}$ has an entirely algebraic character. We can make the same definitions given any group, an element of the centre and a preferred conjugacy class in (in our case the conjugacy class of Dehn twists). So we can ask the same kinds of questions for other groups. In the case of finite permutation groups, with the conjugacy class of transpositions, the problem essentially amounts to the classification of branched covers and was solved by Hurwitz. The definitions are natural with respect to group homomorphisms so one approach to studying the question in the mapping class group might be to consider representations, for example the permutation representation on spin structures. Or one might consider linear representations, the obvious ones being the action on homology which takes one back to the classical homological mondromy. There are also more exotic representations connected with conformal field theory and Jones invrainats, as considered by Smith [11].

Alternatively one can consider "high technology" approaches using Floer homology. This area is developing in a very exciting way through work of Seidel [10] and others, but so far has not yielded any definite results about the classification of symplectic 4-manifolds.

Taking a different direction, there are some fundamental issues left open in the basic theory described above. The main one involves understanding better the nature of asymptotic sequences which we can now formulate as

Problem 2 Show that the inclusion of Proposition 5 is a bijection. This will probably require more thought about the analytical and geometric constructions which underpin the theory.

Another question is suggested by the Auroux-Katzarkov doubling formula. Suppose we know one element $f_{k_{0}}$ of an asymptotic sequence. Can we describe $f_{l}$ for other values of $l$ apart from $l=2^{r} k_{0}$ ? Or perhaps better

Problem 3 Given a topological description of $f_{k_{0}}, f_{k_{1}}$ describe $f_{k_{0}+k_{1}}$.

A good understanding of this would enable one to drop the rather artificial introduction of the subclass $\mathcal{D}_{g, \nu, p}^{(0)}$ in the discussion above.

More generally still, if we have two pencils on a symplectic manifold $X$ with fibre classes $F_{1}, F_{2}$ one can ask for a description of a TLP with fibre $F_{1}+F_{2}$ (if such exists). This might give information about the problem of describing the classes represented by symplectic forms on a fixed 4-manifold.

Rather than trying to use the TLP description to reduce questions to combinatorics one can attempt to use it as a tool to prove general properties of symplectic 4-manifolds. So far, this has been more fruitful, giving a new 
approach to Taubes' results independent of the Seiberg-Witten theory [6], [13], [9]. There is also a generalisation of the TLP description to other 4manifolds [3] and there are many things one could try here; for example to prove that any 4-manifold has "simple type". One thing that should be important to understand is the role of the canonical class $K(\omega)$. Using the Seiberg-Witten theory and pseudo-holomorphic curve techniques, a complete classification is known of symplectic 4-manifolds with $\omega . K(\omega)<0$, i.e. with $\theta<0$ in our notation above. The only examples are the standard ones given by rational and ruled complex surfaces [8]. It would be interesting to derive this by the Lefschtez pencil method:

Problem 4 Reproduce the classification of manifolds with $\omega \cdot K(\omega)<0$ by studying the sets $\mathcal{C}_{\chi, \theta}$ for $\theta<0$.

There is a network of interesting questions dealing with the borderline case when $K(\omega) \cdot \omega=0$ or, stronger still, $K(\omega)=0$. In the latter case the only known examples are the the standard complex tori, certain other torus bundles over tori and K3 surfaces. So we have:

Problem 5 Analyse the monodromy of Lefschetz fibrations on manifolds with $K(\omega)=0$.

Related to this is the general question of understanding the place of complex algebraic surfaces among general symplectic 4-manifolds. One can ask:

Problem 6 Find special features of the monodromy of algebraic surfaces.

There is some good motivation for this coming from at least three directions

- The problem includes (in principle) the well-known problem of describing possible fundamental groups of algebraic surfaces.

- One famous constraint is the "hard Lefschetz" property, which has a well-known translation into the action of the monodromy on homology.

- From the Seiberg-Witten theory we know that there are strong restrictions on the basic classes of algebraic surfaces, and these can be translated into the TLP point of view along the lines of [6], [13].

\section{References}

[1] D. Auroux and L. Katzarkov A degree-doubling formula for braid monodromies and Lefschetz pencils Preprint

[2] D. Auroux, V. Munoz and F. Presas Lagrangian submanifolds and Lefschetz pencils $\mathrm{SG} / 0407126$ 
[3] D. Auroux, S. Donaldson and L. Katzarkov Singular Lefschetz pencils DG/0410332

[4] S. Donaldson Lefschetz pencils on symplectic manifolds J. Differential Geometry 44 (1999) 205-36

[5] S. Donaldson Polynomials, vanishing cycles and Floer homology In: Mathematics: Frontiers and Perspectives (Arnold, Atiyah, Lax, Mazur Eds.) Amer. Math. Soc. 2000, 55-64

[6] S. Donaldson and I. Smith Lefschetz pencils and the canonical class for symplectic four-manifolds Topology 42 (2003) 743-85

[7] R. Gompf and A. Stipsicz Four-manifolds and Kirby calculus Graduate Studies in Mathematics, Amer. Math. Soc. 1999

[8] A-K. Liu Some new applications of general wall-crossing formula, Gompf's conjecture and applications Math. Res. Letters 3 (1996) 569-85

[9] P. Ozsvath and Z.Szabo Holomorphic triangle invariants and the topology of symplectic four-manifolds Duke Math. J. 121 (2004) 1-34 SG/0201049

[10] P. Seidel A long exact sequence for symplectic Floer cohomology Topology 42 (2003) 1003-63 SG/0105186

[11] I. Smith Symplectic four-manifolds and conformal blocks SG/0302088

[12] I. Smith Lefschetz pencils and divisors in moduli space Geometry and Topology 5 (2001) 579-608

[13] I. Smith Serre-Taubes duality for pseudo-holomorphic curves Topology 42 (2003) 937-79 